\title{
Análise de informações técnico-científicas em bulas de medicamentos anticonvulsivantes utilizados na gravidez
}

\author{
Analysis of information in technical-scientific in package \\ inserts of anticonvulsant drugs used in pregnancy
}

\author{
Aníbal de Freitas SANTOS JUNIOR ${ }^{1}$, Caroline de Aragão TANNUS', \\ Jose dos Prazeres ALCANTARA JUNIOR ${ }^{2}$, Edimar CAETITE JUNIOR ${ }^{2}$ \\ ${ }^{1}$ Departamento de Ciências da Vida (DCV), Universidade do Estado da Bahia (UNEB). \\ Rua Silveira Martins, 2555, bairro Cabula. Salvador, BA. CEP: 41150-000. Brasil. \\ ${ }^{2}$ Departamento de Saúde (DSAU), Universidade Estadual de Feira de Santana (UEFS). \\ Av. Transnordestina, s/n, bairro: Novo Horizonte, Feira de Santana, Ba. CEP: 44036-900. Brasil.
} E-mail: edimarcaetite@bol.com.br

\begin{abstract}
The aim of this study was to analyze the technical and scientific information contained in the package inserts of anticonvulsant drugs, as the potential risks and pharmacological changes during pregnancy. Insufficient information regarding the pharmacological procedures and possible risks of four drugs (valproic acid, carbamazepine, phenytoin and diazepam) used as anticonvulsants were analyzed, comparing the data obtained in the inserts of drugs with scientific information disseminated in the research literature and legislation package directions current. Data collection was performed with the investigation of sections Patient Information and Information for Health Professionals of the package inserts obtained in dictionaries pharmaceutical specialties (DEF), official sites of the manufacturers themselves or institutions engaged in health activities. One package inserts, $100 \%$ had absence of the item changes in the pharmacokinetics of antiepileptic drugs during pregnancy; regulation of doses to pregnant women and classification of the drug as the risk category for pregnant women. In the detail, warnings and risks in pregnancy and lactation, $40 \%$ of the inserts analyzed were considered incomplete for not having the potential teratogenic risk to the fetus. The risks of self-medication and treatment interruption were absent in about 33\% of the package inserts and analyzed, about $40 \%$ of these had information about drug interactions with contraceptives. Therefore, the information provided in sections Patient Information and Health Professionals Information (or Technical Information), in their majority, were unsatisfactory as information material for pregnant women, according to the criteria considered.
\end{abstract}

Key Words: anticonvulsants; package inserts; pregnancy

RESUMO:

O objetivo deste trabalho foi analisar as informações técnico-científicas contidas nas bulas de medicamentos anticonvulsivantes, quanto a possíveis riscos e alterações farmacológicas durante a gravidez. Foram analisadas informações, sobre os processos farmacológicos e possíveis riscos de quatro fármacos (ácido valpróico, carbamazepina, diazepam e fenitoína) usados como anticonvulsivantes, confrontando os dados obtidos nas bulas de medicamentos com as informações científicas difundidas na literatura pesquisada e na legislação de bulas vigente. A coleta de dados foi efetuada com a investigação das seções Informações ao Paciente e Informações aos Profissionais de Saúde das bulas obtidas em dicionários de especialidades farmacêuticas (DEF), em sites oficiais dos próprios fabricantes ou de instituições que exercem atividades na área da saúde. Das bulas analisadas, 100\% apresentaram ausência do item alterações na farmacocinética das drogas antiepilépticas durante a gravidez; regulação das doses para gestantes e classificação da droga quanto à categoria de risco para mulheres grávidas. No item, advertências e riscos na gravidez e lactação, $40 \%$ das bulas analisadas foram consideradas incompletas por não apresentarem os possíveis riscos teratogênicos ao feto. Os riscos da automedicação e interrupção do tratamento estavam ausentes em cerca de 33\% das bulas analisadas e, cerca de $40 \%$ destas, apresentavam informações sobre interações medicamentosas com contraceptivos. Portanto, as informações presentes nas seções Informações ao Paciente e Informações ao Profissional da Saúde (ou Informações Técnicas), em sua maioria, foram insatisfatórias como material informativo para as gestantes, segundo os critérios considerados.

Palavras-Chave: anticonvulsivantes; bulas de Medicamentos; gravidez 


\section{INTRODUÇÃO}

O termo convulsão refere-se a uma alteração transitória do comportamento causada pela ativação desordenada, sincrônica e ritmada de populações de neurônios cerebrais, podendo envolver a consciência, função motora, percepção ou sensação (1). O tratamento, na maioria das crises, ainda é realizado com a administração de fármacos anticonvulsivantes. A preferência por certos fármacos varia segundo a tradição e a experiência dos diferentes serviços. Os fatores que influenciam a escolha dos medicamentos são, basicamente, a sua eficácia e efetividade (toxicidade, facilidade de uso, custo, etc). Assim, a avaliação da eficácia envolve dificuldades importantes, inclusive éticas, e medida pela redução de número e intensidade de vários tipos de crises. Essas, entretanto, estão sujeitas a variações multifatoriais que dificultam qualquer análise (2).

A convulsão é um dos problemas neurológicos mais frequentes em gestantes, ocorrendo em 0,3 a $0,6 \%$ das gestações. Porém, a evolução da enfermidade durante a gravidez é bastante variável e imprevisível. Há evidência de exacerbação das crises entre 17 a $37 \%$ das pacientes, uma redução das crises em $50 \%$ das pacientes e nenhuma modificação na frequência das crises nas demais pacientes durante o período gravídico (2-3).

Durante o período gravídico os medicamentos anticonvulsivantes apresentam riscos terapêuticos significantes, tanto para o organismo materno, quanto para o organismo fetal. As possíveis reações adversas ao medicamento são, na maioria das vezes, irreversíveis durante a fase embrionária. Esses efeitos danosos podem determinar malformações congênitas, defeitos morfológicos irreversíveis e outros efeitos bioquímicos e comportamentais no neonato ou em fases mais avançadas da vida. A suspensão do tratamento dessas mulheres apresenta árduos problemas terapêuticos, pois há evidências de que a maioria dos fármacos anticonvulsivantes produza efeitos nocivos ao feto. Neste contexto, a sua suspensão é muito arriscada, tanto para a paciente quanto para o feto, porque podem provocar crises convulsivas na paciente gerando alterações motoras graves e o perigo de hipóxia fetal (4-6).

\section{A United States Food and Drugs Administration} (FDA) classifica os fármacos quanto aos efeitos na gestação em cinco categorias (A, B, C, D, X), também aceitadas aqui no Brasil. Estas categorias abrangem todos os fármacos de absorção sistêmica ou aqueles que apresentam risco potencial para o feto. Estas indicações devem estar inclusas em todas as bulas de medicamentos destinados a esse grupo de pacientes, como determinou a Resolução (RE) $\mathrm{n}^{\circ} 1.548$ da Anvisa, 23 de setembro de 2003, que traz a classificação dos fármacos quanto ao seu risco para as gestantes, a sua obrigatoriedade de ser publicada em anexo nas bulas de medicamentos e um guia para frases de alerta específica para cada grupo de fármaco presente nesta classificação, também obrigatório nas bulas dos medicamentos (7).
O medicamento, sob o ponto de vista legal, é um produto de consumo e o paciente tem o direito a receber todas as informações necessárias para a sua adequada utilização e conservação (8). A informação é parte integrante do medicamento, imprescindível ao seu emprego terapêutico (9). No Brasil, a bula representa o principal material informativo fornecido aos pacientes na aquisição de medicamentos produzidos pelas indústrias farmacêuticas. Os medicamentos produzidos no Brasil e, também, os importados, segundo a legislação vigente, têm a obrigatoriedade de incluir a bula na embalagem dos medicamentos. O roteiro de normatização para a construção dos textos das bulas de medicamentos foi introduzido, pela Portaria $\mathrm{n}^{\mathrm{o}} 65$, da Secretaria Nacional de Vigilância Sanitária (SNVS), de 28 de dezembro de 1984 (10), em vigor até março de 1997, que foi substituída pela Portaria $\mathrm{n}^{\circ}$ 110, da Secretaria de Vigilância Sanitária (SVS), de 18 de março de 1997 (11). Esta última portaria foi substituída, recentemente, pela Resolução da Diretoria Colegiada (RDC) $\mathrm{n}^{\circ}$ 140, de 29 de maio de 2003 (12), publicada no Diário Oficial da União (DOU), a qual determina, atualmente, o conteúdo e organização das bulas de medicamento para pacientes e profissionais de saúde.

Comparando a RDC 140/2003 com a antiga legislação que preconizava o conteúdo das bulas, a Portaria $\mathrm{n}^{\mathrm{o}} 110 / 1997$, ocorreram algumas modificações, podendo ser citadas três novidades importantes: o aumento das letras das bulas, com um tamanho mínimo de $1,5 \mathrm{~mm}$; a seção “Informações ao Paciente” escrita em linguagem acessível e de fácil compreensão e a elaboração de uma bula específica destinada aos profissionais da saúde para os medicamentos de uso hospitalar (13). Ainda de acordo com essa resolução, as bulas, devem conter uma seção específica destinada à informação dirigidas aos pacientes, além das seções de "Identificação do Produto", "Informação Técnica” e "Dizeres Legais" (14).

Atualmente, a RDC 140/2003 foi revogada pela RDC 47/2009 (15) que estabelece regras para elaboração, harmonização, atualização, publicação e disponibilização de bulas de medicamentos para pacientes e para profissionais de saúde. Esta Resolução estabelece diretrizes para bulas dos medicamentos biológicos, específicos, dinamizados, fitoterápicos, notificados, genéricos, similares e novos. Ainda, a RDC 47/2009 discute alguns itens que não foram tratados ou não estavam claros na RDC $n^{\circ} 140 / 2003$, como por exemplo, a apresentação textual e a determinação da presença de bula em formato especial para publicações, e bulas por meio eletrônico e para portadores de deficiência visual. Outro aspecto importante da RDC 47/2009 é a atenção quanto aos efeitos adversos; portanto, esta Resolução propôs que a frequência dos efeitos adversos deva ser ordenada das mais comuns para as mais raras.

Em se tratando de uma parte fundamental, a orientação do uso do medicamento, que permita alcançar o êxito do seu uso racional e em particular 
por meio da bula, faz-se necessário o conhecimento específico e atualizado sobre o medicamento pelo prescritor, farmacêutico assistente e pela indústria farmacêutica, que em suas escalas específicas são responsáveis por dispor de forma clara e objetiva as informações ao paciente, para que este compreenda e adira ao tratamento (16).

A apresentação gráfica do conteúdo informacional nas bulas de medicamento influencia sua leitura e compreensão, e, portanto o uso dos medicamentos. Diferentes tipos de informação contidos na bula, como advertências, tabelas, diagramas e termos específicos da área de saúde, tornam-nas um documento de considerável complexidade linguística e gráfica para os pacientes/ usuários com pouca ou nenhuma experiência de leitura sobre este tipo de conteúdo $(17,18)$.

Devido à escassez de trabalhos envolvendo anticonvulsivantes, com esta proposta, este artigo teve como objetivo analisar as informações técnico-científicas de medicamentos anticonvulsivantes (ácido valpróico, carbamazepina, diazepam e fenitoína), quanto a possíveis riscos e alterações farmacológicas durante a gravidez, enfocando suas influências nos processos farmacocinéticos em grávidas e seus potenciais riscos para o feto.

\section{MÉTODO}

O trabalho foi realizado por meio da análise e interpretação das informações dos textos das bulas de medicamentos anticonvulsivantes comercializados no Brasil. Foram avaliadas as informações contidas nas bulas, referentes aos aspectos farmacológicos e potenciais riscos durante a gravidez. Posteriormente, os dados obtidos foram comparados com as informações descritas na literatura científica (livros didáticos de farmacologia e psicofarmacologia, jornais, periódicos, artigos científicos, monografia dos fármacos e a legislação de bulas de medicamentos no Brasil. Ainda, foram buscadas informações com auxílio da internet em sites específicos na área de saúde, tais como Pubmed, Medline, Scielo, Sciencedirect, etc. Os descritores utilizados foram: Anticonvulsivantes, Bulas de Medicamentos, Informação e Gravidez.

Os textos das bulas de medicamentos foram analisados com base na Resolução RDC 47/2009, por meio dos itens relativos à Identificação do Medicamento, Informações Técnicas aos Profissionais de Saúde e Dizeres Legais. As bulas foram obtidas em dicionários de especialidades farmacêuticas (DEF), em sites oficiais dos próprios fabricantes e instituições que exercem atividades na área da saúde. A amostra constou de 15 bulas dos 04 fármacos selecionados, independentes de serem medicamentos de referência, similares ou genéricos: ácido valpróico, 04 bulas; carbamazepina, 02 bulas; diazepam, 06 bulas; fenitoína, 04 bulas.
A coleta de dados das bulas foi efetuada com a investigação das seções Informações ao Paciente e Informações aos Profissionais de Saúde, sendo propostos seis itens de avaliação correlacionados com as informações sobre a farmacocinética e o uso dos fármacos durante o período gravídico, formulando-se os seguintes itens propostos pela importância para as gestantes, que compõem um grupo com condições clínicas especiais:

1) Alterações na farmacocinética das drogas antiepilépticas durante a gravidez;

2) Advertências e riscos na gravidez e lactação;

3) Riscos da automedicação e interrupção do tratamento;

4) Regulação das doses para gestantes;

5) Classificação da droga quanto à categoria de risco para mulheres grávidas;

6) Interação medicamentosa com contraceptivos orais (proposto para mulheres não grávidas que fazem uso da terapia antiepiléptica, de forma a evitar gravidez inesperada e de risco).

Segundo a metodologia empregada por Gonçalves et al. (2002), para a avaliação da adequação dos textos das bulas de medicamentos comercializados no Brasil (19), os itens propostos acima foram analisados quanto à presença ou ausência de informação, sendo estes classificados como: informação correta, incompleta, incorreta ou ausente; e quanto ao grau de satisfação das bulas.

Os dados oriundos da avaliação foram agrupados por fármacos e a frequência relativa foi calculada, dos itens corretos incompletos, incorretos e ausentes para cada item analisado. Partindo-se desta avaliação, foi proposto um grau de satisfação para as bulas como material informativo para as gestantes:

- Totalmente satisfatórias (todos os itens propostos corretos);

- Satisfatória (04 a 05 itens corretos e nenhum incorreto);

- Parcialmente satisfatório (02 a 03 itens corretos e nenhum incorreto);

- Insatisfatória (menos que 02 itens corretos ou pelo menos um incorreto);

\section{DISCUSSÃO}

Os resultados encontrados após análise dos seis itens de avaliação correlacionados com as informações sobre a farmacocinética e o uso dos fármacos anticonvulsivantes durante o período gravídico, propostos anteriormente para a investigação das seções Informações ao Paciente e Informações aos Profissionais de Saúde das bulas pesquisadas, estão demonstrados na Tabela 01 . 
Tabela 01. Distribuição (em porcentagem) da análise das bulas de anticonvulsivantes na gravidez, quanto às informações presentes

\begin{tabular}{|l|c|c|c|c|}
\multicolumn{1}{|c|}{ ITENS AVALIADOS } & \multicolumn{3}{|c|}{ INFORMAÇÕES NAS BULAS (N=15) } \\
\cline { 2 - 4 } & Correta & Incompleta & Incorreta & Ausente \\
\hline Alterações na farmacocinética de fármacos antiepilépticos durante a gravidez & 0 & 0 & 0 & 100 \\
\hline Advertências e riscos na gravidez e lactação & 53,33 & 40 & 0 & 6,67 \\
\hline Riscos da automedicação e interrupção do tratamento & 46,67 & 20 & 0 & 33,33 \\
\hline Regulação das doses para gestantes & 0 & 0 & 0 & 100 \\
\hline Classificação do fármacos quanto à categoria de risco para mulheres grávidas & 0 & 0 & 0 & 100 \\
\hline Interação medicamentosa com contraceptivos orais & 40 & 0 & 0 & 60 \\
\hline
\end{tabular}

Das bulas analisadas, $100 \%$ apresentaram ausência do item alterações na farmacocinética das drogas antiepilépticas durante a gravidez, porém foram observadas informações sobre a alteração da farmacocinética em outras condições clínicas especiais como em recém-nascidos, idosos, pacientes com doenças hepáticas e com insuficiência renal.

Paralelo à análise das bulas dos medicamentos, foi realizada uma pesquisa das principais alterações fisiológicas que resultam em diferenças na farmacocinética materna dos fármacos administrados durante a gravidez, comparativamente, ao estado não-gravídico. As tabelas 02 e 03 mostram as principais alterações sobre os principais parâmetros farmacocinéticos durantes a gravidez. Essas variáveis farmacocinéticas modificadas acabam por determinar as respostas terapêuticas tóxicas e fatais aos fármacos, e seus metabólitos ou compostos tóxicos, na circulação materna capazes de atravessar a placenta (20).

Gonçalves et al. (2002) (19) destacaram que o item das bulas "características farmacológicas" de- veria conter informações técnicas importantes, envolvendo a farmacocinética, para os profissionais de saúde por permitir correlacionar todas as variáveis envolvidas no emprego do fármaco.

Com relação ao item "advertências e riscos na gravidez e lactação", $40 \%$ das bulas analisadas foram consideradas incompletas por não apresentarem os possíveis riscos teratogênicos ao feto e $6,67 \%$ das mesmas não apresentaram este item. Além de parâmetros farmacocinéticos, alguns fármacos administrados à lactante podem ser excretados pelo leite materno, serem absorvidos pelo lactente e produzir efeitos indesejáveis (9). Ainda neste contexto, alguns fatores podem reduzem a capacidade da mãe de metabolizar ou excretar o fármaco, podendo, desta forma, aumentar a exposição do lactente ao mesmo. Neste trabalho, os autores consideram o ácido valpróico, a carbamazepina, a fenitoína, a fosfenitoína e o sulfato de magnésio seguros para uso durante a lactação (21).

Tabela 02. Principais alterações farmacocinéticas observadas durante a gravidez.

\begin{tabular}{|c|c|c|}
\hline PROCESSOS FARMACOCINÉTICOS & PARÂMETROS FARMACOCINÉTICOS & ALTERAÇÕES \\
\hline \multirow{2}{*}{ Absorção } & Esvaziamento Gástrico & $\downarrow$ \\
& Motilidade Intestinal & $\downarrow$ \\
\hline & Nivel de Progesterona & $\uparrow$ \\
\hline \multirow{2}{*}{ Distribuição } & pH Gástrico & $\uparrow$ \\
\hline & Tmáx & $\downarrow$ \\
\hline Biotransformação & Cmáx & $\uparrow$ \\
\hline & Vd Aparente & $\downarrow$ \\
\hline Eliminação & Concentração de Albumina & $\downarrow$ \\
& Ligação à Proteína Sérica & $\uparrow$ \\
\hline & Atividade do Sistema Enzimático P450 & $\uparrow$ \\
\hline
\end{tabular}


O item "riscos da automedicação e interrupção do tratamento" estava correto em $46,67 \%$, ausente em $33,33 \%$ e incompleto em $20 \%$ das bulas analisadas. Nas bulas consideradas incompletas, a principal razão para a classificação foi a ausência dos riscos da interrupção do tratamento para a paciente. Em seus estudos, Silva et al. (2000) destacaram poucas informações sobre as consequências da interrupção abrupta ou gradual do medicamento (8).

Quanto ao item "regulação das doses para gestantes", $100 \%$ das bulas apresentaram ausência deste item, ocorrendo o mesmo com o item "alterações na farmacocinética das drogas antiepilépticas durante a gravidez", o qual tem como objetivo apresentar alterações posológicas para pacientes com condições clínicas especiais como em recém-nascidos, pacientes idosos, com doenças hepáticas e com insuficiência renal, sem nenhuma menção à posologia especial para gestantes. A monoterapia e as menores doses efetivas devem ser, sempre que possível, o objetivo da terapia anticonvulsivante nas mulheres epilépticas grávidas (3)

Tabela 03. Principais alterações farmacocinéticas em fármacos anticonvulsivantes. durante a gravidez

\begin{tabular}{|c|c|c|c|c|}
\hline FÁrMACO & $\begin{array}{c}\text { CONCENTRAÇÃO } \\
\text { PLASMÁTICA }\end{array}$ & CLEARANCE & MEIA-VIDA & VOLUME DE DISTRIBUIÇÃo \\
\hline Ácido Valpróico & $\downarrow$ & $\uparrow$ & - & $\uparrow$ \\
\hline Carbamazepina & $\downarrow$ & $\uparrow$ & IIIIIIIIIIIIIIIIII- & - \\
\hline Diazepam & $\uparrow$ & - & - & - \\
\hline Fenitoína & $\downarrow$ & $\uparrow$ & - & - \\
\hline
\end{tabular}

Fonte: Silva \& Barros, 1998

O uso prolongado, em altas doses, de benzodiazepínicos em gestantes deve ser evitado, pois pode resultar na acumulação no recém-nascido, e associar-se ao desenvolvimento da síndrome do "floppy baby" e/ ou de abstinência. Ainda, destacam que o uso de doses elevadas, e períodos prolongados de valproato de sódio pode associar-se a sintomas extrapiramidais no recém-nascido, podendo este vir a apresentar hiperatividade, movimentos anormais e tremores (19).

Para o item "classificação da droga quanto à categoria de risco para mulheres grávidas", $100 \%$ das bulas apresentaram uma lacuna, apesar de ter sido determinada a obrigatoriedade da publicação das categorias de risco de fármacos destinados a mulheres grávidas e a presença das frases de alerta quanto à categoria do fármaco pela RE 1548/2003 (7). Estas informações são importantes para as gestantes e para os profissionais da saúde para uma melhor conduta da terapêutica e uso do medicamento. A maioria dos anticonvulsivantes é classificada como risco C ou D, na escala proposta pelo FDA. Apesar da carbamazepina e a oxcarbazepina apresentarem risco C, tornam-se melhores opções entre os anticonvulsivantes, particularmente para o primeiro trimestre, quando comparadas ao valproato/divalproato, que atravessam facilmente a barreira hematoplacentária, podendo provocar malformações, em particular a espinha bífida, pois são classificados com risco D (22).

$\mathrm{O}$ item "interação medicamentosa com contraceptivos orais" foi apresentado de forma correta em $40 \%$ das bulas, nas quais foram citadas várias intera- ções medicamentosas sem alguma menção aos contraceptivos esteroides orais, mesmo que essa informação seja importante para mulheres epilépticas em vigência no tratamento, com possibilidade de engravidar, pois os fármacos anticonvulsivantes podem reduzir a eficácia desse método contraceptivo, aumentando o risco de uma gravidez inesperada podendo ser perigosa para o feto, em vista do tratamento medicamentoso da epilepsia (23).

Quanto ao grau de satisfação atribuída às bulas analisadas como material informativo para as gestantes, os resultados encontrados foram: $46,67 \%$ das bulas apresentaram-se parcialmente satisfatórias e 53,33\% insatisfatória, sendo os itens "alterações na farmacocinética das drogas antiepilépticas durante a gravidez", "regulação das doses para gestantes" e "classificação da droga quanto à categoria de risco para mulheres grávidas", os que mais influenciaram no grau de insatisfação das bulas, por serem ausentes em 100\% delas.

Após a observação dos resultados obtidos neste trabalho, vários foram os possíveis fatores da não conformidade das bulas, entre eles destacando-se o papel da Indústria Farmacêutica e do Sistema de Vigilância Sanitária no Brasil, que vem implementando com maior importância o material técnico informativo, com a criação de uma lista padrão de medicamentos, onde são citadas as especialidades farmacêuticas de referência em bulas, como pode ser observado no bulário eletrônico, criado pela própria ANVISA em maio de 2005 e, disponível em seu endereço eletrônico. 
A bula é quase que o único material informativo ao alcance dos pacientes e desta maneira, poderia suprir, em parte, a carência de uma assistência farmacêutica adequada quanto à utilização do medicamento e da facilitação da automedicação promovida pelas bulas irregulares. Desta maneira é de grande importância informar ao médico, farmacêutico e demais profissionais da saúde sobre a ocorrência de gravidez durante o uso de fármacos antiepilépticos, principalmente para aqueles que apresentam crises convulsivas de alto risco. Estas medidas preventivas são relevantes para que a paciente possa ter uma melhor monitorização do seu tratamento medicamentoso, apresentando um menor risco para o feto e uma gravidez sem maiores complicações.

\section{CONCLUSÃO:}

Conhecer a ação terapêutica, as reações adversas e o comportamento que o fármaco apresenta dentro do organismo são instrumentos muito importantes para se tomar decisões terapêuticas mais racionais e menos prejudiciais para os pacientes.

Observando a análise das bulas dos medicamentos, ficou evidenciado que as informações presentes nas seções Informações ao Paciente e Informações ao Profissional da Saúde (ou Informações Técnicas), em sua maioria, foram insatisfatórias como material informativo para as gestantes, segundo os critérios considerados. Este grau de insatisfação foi decorrente da não inclusão de informações relevantes para as gestantes como: alterações na farmacocinética das drogas antiepilépticas durante a gravidez, regulação das doses para gestantes e classificação da droga quanto à categoria de risco para mulheres grávidas, e da não uniformidade das informações contidas nas bulas entre os medicamentos contendo o mesmo fármaco (na mesma forma farmacêutica), onde a omissão dos dados foi a sua principal causa.

\section{REFERÊNCIAS}

1. McNamara JO. Fármacos eficazes no tratamento das epilepsias. In: Brunton LL, Chabner BA, Knollmann BC. As bases farmacológicas da terapêutica de Goodman \& Gilman. 12.ed. Porto Alegre: AMGH Editora Ltda. 2012. p.583-607.

2. Bragatti JA, Rotta FT. Antiepilépiticos. In: Fuchs FD, Wannmacher L. Farmacologia Clínica: fundamentos da terapêutica racional. 4.ed. Rio de Janeiro: Guanabara Koogan, 2010. p.688-710.

3. Lorenzato RZ, Cavalli RC, Duarte G, Sakamoto AC, Mauad Filho F, Nogueira AA, Cunha SP. Epilepsia e gravidez: evolução e repercussões. Rev. Bras. Ginecol. Obstet. $2002 ; 24(8): 521-526$.

4. Silva LR. Farmacologia pediátrica. In: Silva P. Farmacologia. 8.ed. Rio de Janeiro: Guanabara Koogan. 2010. p.1164-1179.

5. Pennell PB. Pregnancy in the Woman with Epilepsy: Maternal and Fetal Outcomes. Semin Neurol. 2002; 22(3):299-307.

6. Olafsson E, Hallgrimsson JT, Hauser WA, Ludvigsson P, Gudmundsson G. Pregnancies of Women with Epilepsy: A Population-Based Study in Iceland. Epilepsia. 1998; 39(8):887-892.

7. BRASIL. Ministério da Saúde. Agência Nacional de Vigilância Sanitária. Resolução RE n 1548 de 23.09.2003d. Dispõe sobre as categorias de risco de fármacos as mulheres grávidas. [citado 2014 jan 20] Disponível em: http:// www. anvisa.gov.br/e-legis.

8. Silva T, Dal-Pizzol F, Bello CM, Mengue SS, Schenkel EP. Bulas de medicamentos e a informação adequada ao paciente. Rev. Saude Publica. 2000; 34(2):184-189.
9. Ribeiro MSS, Nunes RN, Silva CDC, Sudo EC, Mota DM, Coelho HLL. Medicamentos de Risco para a Gravidez e Lactação Comercializados no Brasil: uma Análise de Bulas. Acta Farm. Bonaerense. 2005; 24(3):441-8.

10. BRASIL. Ministério da Saúde. Secretaria Nacional de Vigilância Sanitária. Portaria n. ${ }^{\circ}$ 65/SNVS de 28 de dezembro de 1984. Diário Oficial da União, Brasília (DF), 31 de dezembro de 1984. Seção 1. p.19931.

11. BRASIL. Ministério da Saúde. Secretaria de Vigilância Sanitária. Portaria n. ${ }^{\circ}$ 110/SVS de 10 de março de 1997. Diário Oficial da União, Brasília (DF), 18 de março de 1997. Seção 1. p. 5332.

12. BRASIL. Ministério da Saúde. Agência Nacional de Vigilância Sanitária. Resolução RDC n 140 de 29.05.2003c. Dispõe sobre o texto para confecção da bula de medicamentos. [citado 2014 jan 20] Disponível em: http://www. anvisa. gov.br/e-legis.

13. Silva MCO. Forma e conteúdo das bulas: o que mudou. Fármacos \& Medicamentos. 2004; 28(5):44-48.

14. BRASIL. Ministério da Saúde. Agência Nacional de Vigilância Sanitária (ANVISA). Bulário Eletrônico da ANVISA. Brasília, 2013. Disponível em: http://www.anvisa.gov. br/datavisa/fila_bula/index.asp. Acesso em: 28 fev. 2014.

15. BRASIL. Ministério da saúde. Agência Nacional de Vigilância Sanitária. Resolução de Diretoria Colegiada n 47 , de 08 de setembro de 2009. Estabelece regras para elaboração, harmonização, atualização, publicação e disponibilização de bulas de medicamentos para pacientes e para profissionais de saúde. [citado 2014 mai 20] Disponível em: www.anvisa.gov.br/medicamentos/bulas/rdc_47.pdf. 
16. Silva M, Almeida AE, Oliveira AM, Correia CC, Benzatti FP, Fernandes JT, Barbosa GR, Pimenta CP, Costa TMM, Doneida VC. Estudo da bula de medicamentos: uma análise da situação. Rev. Ciênc. Farm. Básica Apl. 2006; 27(3):229-236

17. Caldeira TR, Neves ERZ, Perini E. Evolução histórica das bulas de medicamentos no Brasil. Cad. Saúde Pública [online]. 2008; 24(4):737-743.

18. Fujita PL, Spinillo CG. Design da informação em bulas de medicamento: análise e classificação da estrutura e apresentação gráfica de seu conteúdo textual. Rev Bras Desig Inform. 2008; 5(3):1-12.

19. Gonçalves AS, Melo G, Tokarski MHL, Barbosa-Branco A. Bulas de medicamentos como instrumento de informação técnico-científica. Rev. Saude Publica. 2002; 36(1):33-39.
20. Ward RM, Mirkin BL. Farmacologia Perinatal/neonatal. In: Brody TM, Larner J, Minneman KP, Neu HC. Farmacologia Humana: da molecular à clínica. 2. ed. Rio de Janeiro: Guanabara Koogan. 1997. p. 249-258.

21. Chaves RG, Lamounier JA, César CC. Medicamentos e amamentação: atualização e revisão aplicadas à clínica materno-infantil. Rev Paul Pediatr, 2007; 25(3):276-88.

22. Costa C, Reis C, Coelho R. Uso de psicofármacos na gravidez. Acta Obstet Ginecol Port; 2010. 4(2):101111.

23. Bachmann KA, Lewis JD, Fuller MA, Bonfiglio MF. Interações Medicamentosas: o novo padrão de Interações medicamentosas e Fitoterápicas. 2.ed. Barueri/SP: Manole, 2006. 